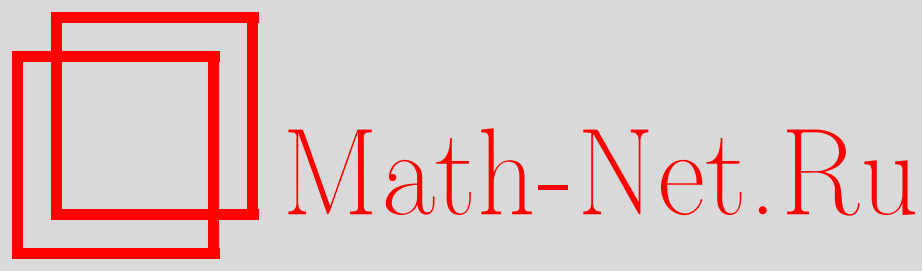

Н. М. Боголюбов, К. Л. Малышев, Корреляционные функции $X X$-магнетика Гейзенберга и случайные блуждания недружественных пешеходов, ТМФ, 2009, том 159, номер 2, 179-193

DOI: https://doi.org/10.4213/tmf6341

Использование Общероссийского математического портала Math-Net.Ru подразумевает, что вы прочитали и согласны с пользовательским соглашением http://www . mathnet.ru/rus/agreement

Параметры загрузки:

IP : 54.224 .60 .19

26 апреля 2023 г., 12:03:19

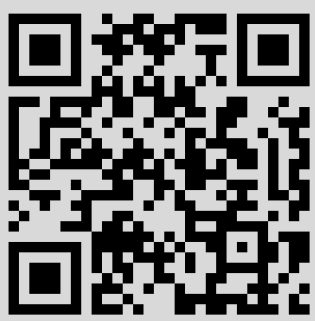




\title{
ФИЗИКА
}

Том 159, № 2

май, 2009

2009 г.

Н. М. Боголюбов*, К. Л. Малышев*

\section{КОРРЕЛЯЦИОННЫЕ ФУНКЦИИ $X X$-МАГНЕТИКА ГЕЙЗЕНБЕРГА И СЛУЧАЙНЫЕ БЛУЖДАНИЯ НЕДРУЖЕСТВЕННЫХ ПЕШЕХОДОВ}

\begin{abstract}
Исследуется связь между случайными блужданиями по одномерной периодической решетке и корреляционными функциями спиновой $X X$-цепочки Гейзенберга. Операторные средние над ферромагнитным состоянием играют роль производящих функций для числа путей случайных блужданий так называемых "недружественных" пешеходов (при встрече в любом из узлов решетки недружественные пешеходы уничтожают друг друга). Показано, что парную корреляционную функцию спинов, вычисленную по всем собственным состояниям $X X$-магнетика, можно интерпретировать как производящую функцию блужданий одиночного пешехода в среде с переменным числом недружественных соседей. Получены ответы для числа путей указанного пешехода, перемещающегося из некоторого фиксированного узла в другой достаточно удаленный узел решетки. Представлены асимптотические оценки числа путей в случае, когда количество выполняемых шагов велико.
\end{abstract}

Ключевые слова: случайные блуждания, магнетик Гейзенберга, корреляционные функции.

\section{1. ВВЕДЕНИЕ}

Случайные блуждания являются классической проблемой как для комбинаторики, так и для статистической физики. Задача о перечислении путей, пройденных так называемыми недружественными пешеходами в результате блужданий по одномерной решетке, была сформулирована и подробно рассмотрена Фишером [1]. Предполагается, что при встрече в одном из узлов решетки любая пара недружественных пешеходов уничтожает не только друг друга, но и всех остальных пешеходов. Указанная задача продолжает привлекать значительное внимание физиков и математиков [2]-[9]. Близкие проблемы возникают также при изучении самоорганизующейся критичности [10], доме́нных стенок [11] и полимеров [12]. В работе [13]

${ }^{*}$ Санкт-Петербургское отделение Математического института им. В. А. Стеклова РАН, Санкт-Петербург, Россия. E-mail: bogoliub@pdmi.ras.ru, malyshev@pdmi.ras.ru 
рассматривается подход к описанию случайного блуждания аннигилирующих частиц по кольцу. В работе [14] изучаются блуждания со случайными поворотами на полуоси, в начале которой могут рождаться частицы.

В работах [15], [16] было показано, что корреляционные функции $X X$-цепочки Гейзенберга над ферромагнитным состоянием могут быть использованы для перечисления путей блуждания недружественных пешеходов. В предлагаемой статье рассмотрены средние специального вида как над ферромагнитным состоянием, так и над суперпозицией собственных состояний $X X$-магнетика в нулевом магнитном поле. Рассмотренные средние играют роль производящих функций числа путей недружественных пешеходов. Вычисление корреляционных функций производится с помощью функционального интегрирования [17], [18]. Получены ответы для числа путей одиночного пешехода, перемещающегося из фиксированного узла в другой достаточно удаленный узел решетки. Представлены асимптотические оценки числа путей в пределе бесконечно большого количества выполняемых шагов (и, соответственно, числа случайных поворотов).

Работа организована следующим образом. Гамильтониан модели и вычисление корреляционных функций обсуждаются в разделе 2. Раздел 3 посвящен конкретным вычислениям и соответствующим асимптотическим оценкам. Обсуждение в разделе 4 завершает статью.

\section{2. МОДЕЛЬ И КОРРЕЛЯЦИОННЫЕ ФУНКЦИИ}

Интересующий нас $X X$-магнетик является частным случаем более общей спиновой модели - $X Y$-цепочки Гейзенберга, гамильтониан которой в поперечном магнитном поле $h>0$ задается следующими формулами [19], [20]:

$$
\begin{aligned}
H & =H_{0}+\gamma H_{1}-h S^{z}, \\
H_{0} & \equiv-\sum_{n, m=1}^{M} \Delta_{n m}^{(+)} \sigma_{n}^{+} \sigma_{m}^{-}, \\
H_{1} & \equiv-\frac{1}{2} \sum_{n, m=1}^{M} \Delta_{n m}^{(+)}\left(\sigma_{n}^{+} \sigma_{m}^{+}+\sigma_{n}^{-} \sigma_{m}^{-}\right), \quad S^{z} \equiv \frac{1}{2} \sum_{n=1}^{M} \sigma_{n}^{z},
\end{aligned}
$$

где $S^{z}$ означает $z$-компоненту оператора полного спина, $\gamma$ - параметр анизотропии. Локальные спиновые операторы $\sigma_{n}^{ \pm}=\left(\sigma_{n}^{x} \pm i \sigma_{n}^{y}\right) / 2$ и $\sigma_{n}^{z}$ представляют собой матрицы Паули, зависящие от решеточного аргумента $n \in \mathcal{M} \equiv\{1,2, \ldots, M\}$, где $M=0(\bmod 2)$. Соответствующие перестановочные соотношения имеют вид $\left[\sigma_{k}^{+}, \sigma_{l}^{-}\right]=\delta_{k, l} \sigma_{l}^{z},\left[\sigma_{k}^{z}, \sigma_{l}^{ \pm}\right]= \pm 2 \delta_{k, l} \sigma_{l}^{ \pm}$. Кроме того, введена матрица перескоков $\Delta^{(s)}$ с матричными элементами

$$
\Delta_{n m}^{(s)} \equiv \frac{1}{2}\left(\delta_{|n-m|, 1}+s \delta_{|n-m|, M-1}\right),
$$

где $\delta_{n, l}$ - символ Кронекера, а $s$ может принимать два значения: $s= \pm$. Мы предполагаем, что наложены периодические граничные условия: $\sigma_{n+M}^{\alpha}=\sigma_{n}^{\alpha}$ для любого 
$n \in \mathcal{M}$. При нулевом значении параметра $\gamma$ гамильтониан $H(1)$ сводится к гамильтониану $X X$-магнетика.

Общее определение зависящих от времени $t$ и температуры $T \equiv 1 / \beta$ корреляционных функций рассматриваемой модели имеет вид

$$
G_{j ; l}^{a b}(t) \equiv \frac{1}{Z} \operatorname{Tr}\left(\sigma_{j}^{a}(0) \sigma_{l}^{b}(t) e^{-\beta H}\right), \quad Z \equiv \operatorname{Tr}\left(e^{-\beta H}\right),
$$

где $\sigma_{l}^{b}(t) \equiv e^{i t H} \sigma_{l}^{b} e^{-i t H}$ и $\operatorname{Tr}$ означает усреднение по всем собственным состояниям гамильтониана $H$. Кроме того, в нормировке используется статистическая сумма $Z$. В работе [21] обсуждалось вычисление корреляторов (3) в базисе собственных функций гамильтониана $X X$-магнетика. Основное внимание при этом уделялось связи корреляционных функций с определителями Фредгольма в термодинамическом пределе. В нашей статье мы будем рассматривать только $X X$-цепочку и через $H$ будем обозначать ее гамильтониан.

Для вычисления средних (3) можно воспользоваться переходом от спиновых переменных к каноническим ферми-переменным $c_{j}, c_{j}^{\dagger}, j \in \mathcal{M}$ [19], [20]. Соответствующее преобразование Йордана-Вигнера таково:

$$
\sigma_{n}^{+}=\left(\prod_{j=1}^{n-1} \sigma_{j}^{z}\right) c_{n}, \quad \sigma_{n}^{-}=c_{n}^{\dagger}\left(\prod_{j=1}^{n-1} \sigma_{j}^{z}\right), \quad n \in \mathcal{M},
$$

где $\sigma_{j}^{z}=1-2 c_{j}^{\dagger} c_{j}$. Периодические граничные условия для спиновых операторов приводят к следующим граничным условиям для фермиевских переменных:

$$
c_{M+1}=(-1)^{\mathcal{N}} c_{1}, \quad c_{M+1}^{\dagger}=c_{1}^{\dagger}(-1)^{\mathcal{N}},
$$

где $\mathcal{N}=\sum_{n=1}^{M} c_{n}^{\dagger} c_{n}$ - оператор полного числа частиц. Гамильтониан $H(1)$ в фермионном представлении принимает вид [19], [20]

$$
H=H^{+} P^{+}+H^{-} P^{-},
$$

где $P^{+}\left(P^{-}\right)$- проекторы на состояния с четным (нечетным) числом фермионов: $P_{+}+P_{-}=\mathbb{I}, P_{+}-P_{-}=(-1)^{\mathcal{N}}$. При этом операторы $H^{ \pm}(6)$ формально идентичны, однако индекс $s= \pm$ указывает на соответствующую конкретизацию условий (5): $c_{M+1}=-s c_{1}, c_{M+1}^{\dagger}=-s c_{1}^{\dagger}$. Иными словами, квадратичные по фермионным переменным операторы $H^{ \pm}$имеют следующее представление:

$$
H^{ \pm}=c^{\dagger} \widehat{H}^{ \pm} c-\frac{M h}{2}, \quad \widehat{H}^{ \pm}=-\hat{\Delta}^{(\mp)}+h \hat{I},
$$

где матрицы $\widehat{H}^{ \pm}$выражены через матрицы перескоков $(2)$ и $\hat{I}$ - единичная матрица. Кроме того, в (7) использованы сокращенные обозначения $c^{\dagger}$ и $c$ для $M$-компонентных строки и столбца, построенных из $c_{n}^{\dagger}, c_{n}, n \in \mathcal{M}$.

В частности, коррелятор (3) для $a=b=z$ в представлении (4) записывается как [18], [22], [23]

$$
G_{j ; l}^{z z}(t)=1-\frac{2}{Z} \operatorname{Tr}\left(c_{j}^{\dagger} c_{j} e^{-\beta H}\right)-\frac{2}{Z} \operatorname{Tr}\left(c_{l}^{\dagger} c_{l} e^{-\beta H}\right)+\frac{4}{Z} \operatorname{Tr}\left(c_{j}^{\dagger} c_{j} e^{i t H} c_{l}^{\dagger} c_{l} e^{-(\beta+i t) H}\right) .
$$


Для его вычисления удобно вести производящий функционал [18]

$$
\mathcal{G} \equiv \mathcal{G}(S, T \mid \lambda, \nu)=\frac{1}{Z} \operatorname{Tr}\left(e^{S} e^{-\lambda H} e^{T} e^{-\nu H}\right),
$$

где $\lambda, \nu$ - комплексные параметры, $\lambda+\nu=\beta$. В соотношении (9) использованы квадратичные операторы $S \equiv c^{\dagger} \widehat{S} c$ и $T \equiv c^{\dagger} \widehat{T} c$, задаваемые с помощью матриц $\widehat{S}=\operatorname{diag}\left\{S_{1}, S_{2}, \ldots, S_{M}\right\}$ и $\widehat{T}=\operatorname{diag}\left\{T_{1}, T_{2}, \ldots, T_{M}\right\}$. Например, последнее слагаемое в правой части (8) получается из (9) следующим образом: оно равно

$$
\lim _{\substack{S_{n}, T_{n} \rightarrow 0 \\ n \in \mathcal{M}}} \lim _{\lambda \rightarrow-i t} \lim _{\nu \rightarrow \beta+i t} \frac{\partial}{\partial S_{j}} \frac{\partial}{\partial T_{l}} \mathcal{G}(S, T \mid \lambda, \nu) .
$$

С учетом (6) след в правой части (9) переписывается так [18]:

$$
\operatorname{Tr}\left(e^{S} e^{-\lambda H} e^{T} e^{-\nu H}\right)=\frac{1}{2}\left(\mathcal{G}_{\mathrm{F}}^{+} Z_{\mathrm{F}}^{+}+\mathcal{G}_{\mathrm{F}}^{-} Z_{\mathrm{F}}^{-}+\mathcal{G}_{\mathrm{B}}^{+} Z_{\mathrm{B}}^{+}-\mathcal{G}_{\mathrm{B}}^{-} Z_{\mathrm{B}}^{-}\right),
$$

где

$$
\begin{aligned}
\mathcal{G}_{\mathrm{F}}^{ \pm} Z_{\mathrm{F}}^{ \pm} & \equiv \operatorname{Tr}\left(e^{S} e^{-\lambda H^{ \pm}} e^{T} e^{-\nu H^{ \pm}}\right), \\
\mathcal{G}_{\mathrm{B}}^{ \pm} Z_{\mathrm{B}}^{ \pm} & \equiv \operatorname{Tr}\left(e^{S} e^{-\lambda H^{ \pm}} e^{T}(-1)^{\mathcal{N}} e^{-\nu H^{ \pm}}\right),
\end{aligned}
$$

и $Z_{\mathrm{F}}^{ \pm}=\operatorname{Tr}\left(e^{-\beta H^{ \pm}}\right), Z_{\mathrm{B}}^{ \pm}=\operatorname{Tr}\left((-1)^{\mathcal{N}} e^{-\beta H^{ \pm}}\right)$. При этом для статистической суммы $Z$ получаем $Z=\left(Z_{\mathrm{F}}^{+}+Z_{\mathrm{F}}^{-}+Z_{\mathrm{B}}^{+}-Z_{\mathrm{B}}^{-}\right) / 2$. В термодинамическом пределе слагаемые в $(11)$ с индексом В взаимно компенсируются, и для вычисления $\mathcal{G}(9)$ достаточно вычислить $\mathcal{G}_{\mathrm{F}}^{ \pm}$.

Рассматриваемое фермиевское представление характеризуется фоковским состоянием $|0\rangle$, одинаковым для $H^{+}$и $H^{-}$таким, что $c_{k}|0\rangle=0, k \in \mathcal{M}$. Соответствующие когерентные состояния над $|0\rangle,|z\rangle \equiv e^{c^{\dagger} z}|0\rangle$ и $\left\langle z^{*}\right| \equiv\langle 0| e^{z^{*} c}$ различны для $H^{+}$и $H^{-}$. Здесь мы используем сокращенные обозначения $z^{*} \equiv\left(z_{1}^{*}, \ldots, z_{M}^{*}\right)$ и $z \equiv\left(z_{1}, \ldots, z_{M}\right)$ для наборов независимых грассмановых параметров $z_{k}, z_{k}^{*}, k \in \mathcal{M}$ (дополнительный индекс \pm для $z^{*}, z$ уместно опустить). Кроме того, $\sum_{k=1}^{M} c_{k}^{\dagger} z_{k} \equiv c^{\dagger} z, \prod_{k=1}^{M} d z_{k} \equiv d z$. Будем вычислять $\mathcal{G}_{\mathrm{F}}^{ \pm} Z_{\mathrm{F}}^{ \pm}$в (12), пользуясь представлением следа в подходе грассманова интегрирования [18]:

$$
\mathcal{G}_{\mathrm{F}}^{ \pm} Z_{\mathrm{F}}^{ \pm}=\int d z d z^{*} e^{z^{*} z}\left\langle z^{*}\left|e^{S} e^{-\lambda H^{ \pm}} e^{T} e^{-\nu H^{ \pm}}\right| z\right\rangle .
$$

Чтобы представить правую часть этого равенства в виде функционального интеграла, введем $L$ новых когерентных состояний $|x(I)\rangle,\left\langle x^{*}(I)\right|$, где $I \in\{1,2, \ldots, L\}$. В качестве индексов здесь выступают $2 L$ наборов $x^{*}(I), x(I)$, каждый из которых образован $M$ независимыми грассмановыми параметрами. Использование разложений единицы позволяет представить правую часть $(13)$ в виде $(L+1)$-кратного интеграла. Введем добавочные переменные, выражающие условие квазипериодичности:

$$
-\widehat{E} x(0)=x(L+1) \equiv z, \quad-x^{*}(L+1)=x^{*}(0) \widehat{E}^{-1} \equiv z^{*},
$$


где $\widehat{E} \equiv e^{\widehat{S}} e^{-\lambda \widehat{H}^{ \pm}} e^{\widehat{T}}$. Устремляя $L$ к бесконечности, приходим к функциональному интегралу на пространстве траекторий $x^{*}(\tau), x(\tau)$, где $\tau \in \mathbb{R}:$

$$
\mathcal{G}_{\mathrm{F}}^{ \pm} Z_{\mathrm{F}}^{ \pm}=\int e^{S} d \lambda^{*} d \lambda \prod_{\tau} d x^{*}(\tau) d x(\tau)
$$

Здесь функционал действия $S \equiv \int L(\tau) d \tau$ выражается через лаганжиан

$$
L(\tau) \equiv x^{*}(\tau)\left(\frac{d}{d \tau}-\widehat{H}^{ \pm}\right) x(\tau)+J^{*}(\tau) x(\tau)+x^{*}(\tau) J(\tau)
$$

где

$$
J^{*}(\tau) \equiv \lambda^{*}\left(\delta(\tau) \hat{I}+\delta(\tau-\nu) \widehat{E}^{-1}\right), \quad J(\tau) \equiv(\delta(\tau) \hat{I}+\delta(\tau-\nu) \widehat{E}) \lambda .
$$

Интегрирование в (15) по вспомогательным грассмановым переменным $\lambda^{*}, \lambda$ гарантирует соблюдение наложенной связи (14), и $\delta$-функции в $J^{*}(\tau), J(\tau)$ редуцируют $\tau \in \mathbb{R}$ к $\tau \in[0, \beta]$. Условия стационарности $\delta S / \delta x^{*}=0, \delta S / \delta x=0$ приводят к регуляризованному ответу [18]

$$
\mathcal{G}_{\mathrm{F}}^{ \pm}=\operatorname{det}\left(\hat{I}+\frac{e^{(\beta-\nu) \widehat{H}^{ \pm}} e^{\widehat{S}} e^{-\lambda \widehat{H}^{ \pm}} e^{\widehat{T}}-\hat{I}}{\hat{I}+e^{\beta \widehat{H}^{ \pm}}}\right) .
$$

Далее, мы подставляем (16) в (10) и переходим в импульсное представление. Данная процедура применима и к остальным корреляторам $G_{j ; l}^{a b}(t)(3), a, b \in\{+,-\}$.

\section{3. СЛУЧАЙНЫЕ БЛУЖДАНИЯ}

Как показано в работах [15], [16], перевороты спинов на одномерной решетке можно ассоциировать со случайными перемещениями пешеходов. Действительно, рассмотрим состояние $X X$-цепочки Гейзенберга, соответствующее ферромагнитному упорядочению $M$ спинов: $|\uparrow\rangle \equiv \bigotimes_{n=1}^{M}|\uparrow\rangle_{n}$ (т.е. все спины направлены "вверх"). Рассмотрим среднее следующего вида:

$$
F_{j ; l}(\lambda) \equiv\left\langle\Uparrow\left|\sigma_{j}^{+} e^{-\lambda H_{0}} \sigma_{l}^{-}\right| \Uparrow\right\rangle,
$$

где $H_{0}$ соответствует нулевому магнитному полю $h=0$ в гамильтониане $(6),(7)$ (для соответствующих матриц $\widehat{H}^{ \pm}(7)$ нижний индекс вводить не будем), $\lambda \in \mathbb{C}$ - “эволюционный" параметр. Направление спина "вверх" (или "вниз") соответствует пустым (или заполненным) узлам. Дифференцируя $F_{j ; l}(\lambda)(17)$ и раскрывая коммутатор $\left[H_{0}, \sigma_{j}^{+}\right]$, мы получаем разностно-дифференциальное уравнение

$$
\frac{d}{d \lambda} F_{j ; l}(\lambda)=\frac{1}{2}\left(F_{j+1 ; l}(\lambda)+F_{j-1 ; l}(\lambda)\right)
$$

(аналогичное уравнение можно получить и для фиксированного индекса $j$ ). Решение данного уравнения определяется налагаемыми граничными условиями по решеточному аргументу, а также условием при $\lambda=0$. 
Среднее $F_{j ; l}(\lambda)$ можно рассматривать как производящую функцию траекторий со случайными поворотами, начинающихся в $l$-м узле и оканчивающихся в $j$-м узле. Действительно, введем обозначение $\mathcal{D}_{\lambda}^{K}$ для оператора $K$-кратного дифференцирования по $\lambda$ в точке $\lambda=0$. Применение $\mathcal{D}_{\lambda}^{K}$ к среднему (17) приводит к ответу

$$
\mathcal{D}_{\lambda}^{K}\left[F_{j ; l}(\lambda)\right]=\left\langle\Uparrow\left|\sigma_{j}^{+}\left(-H_{0}\right)^{K} \sigma_{l}^{-}\right| \Uparrow\right\rangle=\sum_{n_{1}, \ldots, n_{K-1}} \Delta_{j n_{K-1}}^{(+)} \ldots \Delta_{n_{2} n_{1}}^{(+)} \Delta_{n_{1} l}^{(+)}
$$

Правая часть (19) совпадает с матричным элементом $j$-й строки и $l$-го столбца матрицы, равной произведению $K$ матриц перескоков $(2)$, каждая из которых соответствует переходу между двумя ближайшими узлами решетки. Будучи умноженной на $2^{K}$ (что связано с принятой нормировкой матрицы (2)), правая часть (19) дает число траекторий из $K$ шагов между $l$-м и $j$-м узлами. Будем обозначать это число $\left|P_{K}(l \rightarrow j)\right|$.

Пусть $\left|P_{K}\left(l_{1}, \ldots, l_{N} \rightarrow j_{1}, \ldots, j_{N}\right)\right|$ обозначает число путей из $K$ звеньев, проходимых $N$ недружественными пешеходами в модели блужданий со случайными поворотами. Здесь начальные и конечные положения пешеходов на узлах заданы, соответственно, последовательностями $l_{1}>l_{2}>\cdots>l_{N}$ и $j_{1}>j_{2}>\cdots>j_{N}$. Рассмотрим $N$-точечную корреляционную функцию $(N \leqslant M)$

$$
F_{j_{1}, j_{2}, \ldots, j_{N} ; l_{1}, l_{2}, \ldots, l_{N}}(\lambda)=\left\langle\Uparrow\left|\sigma_{j_{1}}^{+} \sigma_{j_{2}}^{+} \ldots \sigma_{j_{N}}^{+} e^{-\lambda H_{0}} \sigma_{l_{1}}^{-} \sigma_{l_{2}}^{-} \ldots \sigma_{l_{N}}^{-}\right| \Uparrow\right\rangle .
$$

Данный коррелятор связан с перечислением допустимых траекторий, проходимых $N$ недружественными пешеходами. Действительно, применение к (20) оператора $\mathcal{D}_{\lambda / 2}^{K}$ приводит к среднему вида $\left\langle\Uparrow\left|\sigma_{j_{1}}^{+} \sigma_{j_{2}}^{+} \ldots \sigma_{j_{N}}^{+}\left(-2 H_{0}\right)^{K} \sigma_{l_{1}}^{-} \sigma_{l_{2}}^{-} \ldots \sigma_{l_{N}}^{-}\right| \Uparrow\right\rangle$. Указанное же среднее дает числа $\left|P_{K}\left(l_{1}, \ldots, l_{N} \rightarrow j_{1}, \ldots, j_{N}\right)\right|$, что можно получить с использованием коммутатора

$$
\left[H_{0}, \sigma_{l_{1}}^{-} \sigma_{l_{2}}^{-} \ldots \sigma_{l_{K}}^{-}\right]=\sum_{k=1}^{K} \sigma_{l_{1}}^{-} \ldots \sigma_{l_{k-1}}^{-}\left[H_{0}, \sigma_{l_{k}}^{-}\right] \sigma_{l_{k+1}}^{-} \ldots \sigma_{l_{K}}^{-}
$$

(при этом дифференцирование по $\lambda / 2$, а не по $\lambda$ позволило нам учесть нормировку матрицы перескоков (2)). Условие того, что пути пешеходов не пересекаются, выражается в обращении в нуль корреляционной функции (20) для любой пары совпадающих индексов $l_{k}$ или $j_{p}$.

Дифференцируя определение (20) по $\lambda$ и применяя соотношение (21), получаем уравнение

$$
\frac{d}{d \lambda} F_{j_{1}, \ldots, j_{N} ; l_{1}, \ldots, l_{N}}(\lambda)=\frac{1}{2} \sum_{k=1}^{N}\left(F_{j_{1}, \ldots, j_{N} ; l_{1}, l_{2}, \ldots, l_{k}+1, \ldots, l_{N}}(\lambda)+F_{j_{1}, \ldots, j_{N} ; l_{1}, l_{2}, \ldots, l_{k}-1, \ldots, l_{N}}(\lambda)\right),
$$

которое было рассмотрено в работе [16] для случая периодичности по решеточному аргументу и при начальном условии $F_{j_{1}, \ldots, j_{N} ; l_{1}, \ldots, l_{N}}(0)=\prod_{m=1}^{N} \delta_{j_{m}, l_{m}}$. Функция

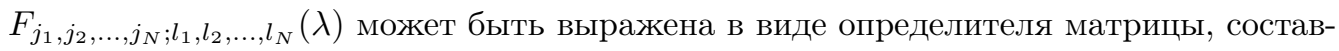
ленной из средних вида (17) [16]:

$$
F_{j_{1}, \ldots, j_{N} ; l_{1}, \ldots, l_{N}}(\lambda)=\operatorname{det}\left(F_{j_{r} ; l_{s}}(\lambda)\right)_{1 \leqslant r, s \leqslant N} .
$$


3.1. Случайные блуждания на оси. Пусть цепочка бесконечна $(M \rightarrow \infty)$. Тогда решением уравнения (18), удовлетворяющим условию $F_{j ; l}(0)=\delta_{j, l}$, оказывается [15] модифицированная функция Бесселя $I_{j-l}(\lambda)$ :

$$
F_{j ; l}(\lambda)=I_{j-l}(\lambda)=\frac{1}{2 \pi} \int_{-\pi}^{\pi} d \theta e^{\lambda \cos \theta} e^{i(j-l) \theta} .
$$

Для $I_{j-l}(\lambda)$ имеется следующее разложение в степенной ряд:

$$
I_{j-l}(\lambda)=\sum_{Q \geqslant|l-j|} \frac{1}{\left(\frac{Q-j+l}{2}\right) !\left(\frac{Q+j-l}{2}\right) !}\left(\frac{\lambda}{2}\right)^{Q}
$$

где индекс суммирования $Q$ подчиняется требованию $Q+|j-l|=0(\bmod 2)$. В пределе больших "времен” $(\lambda \rightarrow \infty)$ и при умеренных $m \equiv|l-j|$ для производящей функции возникает известная асимптотика

$$
F_{j ; l}(\lambda) \simeq \frac{e^{\lambda}}{\sqrt{2 \pi \lambda}}\left(1-\frac{4 m^{2}-1}{8 \lambda}+\cdots\right)
$$

Таким образом, степенное поведение $F_{j ; l}(\lambda)$ определяется показателем $\xi=-1 / 2$.

Пусть число $K$ удовлетворяет соотношениям $K \geqslant|l-j|$ и $K+|j-l|=0(\bmod 2)$. Дифференцируя ряд (25), приходим к биномиальной формуле $\left|P_{K}(l \rightarrow j)\right|=C_{K}^{L}$ для числа всех решеточных путей “длины” $K$ между двумя узлами на бесконечной оси:

$$
\left|P_{K}(l \rightarrow j)\right| \equiv \mathcal{D}_{\lambda / 2}^{K}\left[F_{j ; l}(\lambda)\right]=\frac{(m+2 L) !}{L !(m+L) !} .
$$

Здесь $L \equiv(K-m) / 2$ - половина числа поворотов.

Перейдем к многоточечной корреляционной функции $F_{j_{1}, j_{2}, \ldots, j_{N} ; l_{1}, l_{2}, \ldots, l_{N}}(\lambda)$. Как показано выше, величина $\mathcal{D}_{\lambda / 2}^{K}\left[F_{j_{1}, \ldots, j_{N} ; l_{1}, \ldots, l_{N}}(\lambda)\right]$ имеет смысл числа траекторий $N$ недружественных пешеходов, каждый из которых совершает $K$ шагов. Однако можно дать несколько иную комбинаторную интерпретацию указанной величины. Для этого рассмотрим представление многоточечного коррелятора в виде определителя (23). Для бесконечной цепочки его матричные элементы $F_{j_{r} ; l_{s}}(\lambda)$ являются функциями Бесселя $I_{j_{r}-l_{s}}(\lambda)(24)$. Действие $\mathcal{D}_{\lambda / 2}^{K}$ на определитель будем рассматривать как $K$-кратное дифференцирование произведения $N$ функций:

$$
\left(f_{1}(x) f_{2}(x) \ldots f_{N}(x)\right)^{(K)}=\sum_{n_{1}+n_{2}+\cdots+n_{N}=K} P\left(n_{1}, n_{2}, \ldots, n_{N}\right) f_{1}^{\left(n_{1}\right)} f_{2}^{\left(n_{2}\right)} \ldots f_{N}^{\left(n_{N}\right)} .
$$

В последней формуле использовано обозначение $f^{(n)} \equiv d^{n} f(x) / d x^{n}$, а коэффициентами являются числа $P\left(n_{1}, n_{2}, \ldots, n_{N}\right)$ перестановок с повторениями:

$$
P\left(n_{1}, n_{2}, \ldots, n_{N}\right) \equiv \frac{\left(n_{1}+n_{2}+\cdots+n_{N}\right) !}{n_{1} ! n_{2} ! \ldots n_{N} !} .
$$

Суммирование в правой части равенства (27) распространяется на все неотрицательные значения $n_{1}, n_{2}, \ldots, n_{N}$, сумма которых равна $K$. 
Далее, пусть задана бесконечная (гипер)кубическая решетка размерности $N$, каждый узел которой пронумерован набором из $N$ чисел. Введем обозначение $\mathcal{T}_{K}\left(q_{1}, q_{2}, \ldots, q_{N}\right)$ для числа решеточных траекторий, по которым некоторый пешеход, расположенный в "начальной" точке $\boldsymbol{O} \equiv(0,0, \ldots, 0)$, может переместиться в точку $\left(q_{1}, q_{2}, \ldots, q_{N}\right)$, делая $K$ шагов (за шаг пешеход смещается в один из ближайших узлов). Предположим, что все $q_{k}$ неотрицательны, и выполнено неравенство $K \geqslant q_{1}+q_{2}+\cdots+q_{N}$. Иными словами, возможны шаги, компенсирующие друг друга. Обозначим их количество через $2 L$,

$$
L \equiv \frac{K-q_{1}-q_{2}-\cdots-q_{N}}{2} .
$$

С учетом (29) мы имеем формулу для числа путей:

$$
\mathcal{T}_{K}\left(q_{1}, q_{2}, \ldots, q_{N}\right)=\sum_{L_{1}+L_{2}+\cdots+L_{N}=L} P\left(q_{1}+L_{1}, q_{2}+L_{2}, \ldots, q_{N}+L_{N}, L_{1}, L_{2}, \ldots, L_{N}\right),
$$

где суммирование распространяется на все неотрицательные $L_{1}, L_{2}, \ldots, L_{N}$, сумма которых равна $L$, и используется формула для числа перестановок с повторениями (28).

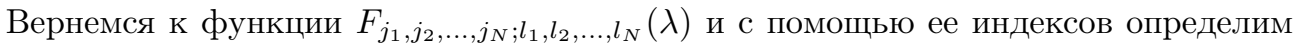
матрицу $\left(n_{r s}\right)_{1 \leqslant r, s \leqslant N}$ с матричными элементами $n_{r s} \equiv j_{r}-l_{s}$. Тогда имеет место следующее

УтвеРЖДЕНИЕ. Число траекторий из $K$ звенъев, проходимых $N$ недружественными пешеходами на оси, выражается через число траекторий такой же "длины” К одиночного пешехода, перемещающегося по узлам $N$-мерной бесконечной решетки:

$$
\begin{aligned}
\left|P_{K}\left(l_{1}, \ldots, l_{N} \rightarrow j_{1}, \ldots, j_{N}\right)\right| & \equiv \mathcal{D}_{\lambda / 2}^{K}\left[F_{j_{1}, \ldots, j_{N} ; l_{1}, \ldots, l_{N}}(\lambda)\right]= \\
& =\sum_{S_{a_{1}, a_{2}, \ldots, a_{N}}}(-1)^{\mathcal{P}_{S}} \mathcal{T}_{K}\left(n_{a_{1} 1}, n_{a_{2} 2}, \ldots, n_{a_{N} N}\right),
\end{aligned}
$$

где суммирование идет по всем перестановкам $S_{a_{1}, a_{2}, \ldots, a_{N}} \equiv S\left(\begin{array}{c}1,2, \ldots, N \\ a_{1}, a_{2}, \ldots, a_{N}\end{array}\right)$ чисел $1,2, \ldots, N$, u $\mathcal{P}_{S}$ есть четность конкретной перестановки.

ДокАзАтЕльство. Чтобы получить равенство (31), следует разложить определитель (23) по строке или по столбцу и применить индукцию, используя при этом соотношения (26)-(30).

Вычислим (30), например, для $N=2$ :

$$
\mathcal{T}_{K}\left(q_{1}, q_{2}\right)=C_{q_{1}+q_{2}+2 L}^{q_{1}+L} \sum_{k=0}^{L} C_{q_{1}+L}^{L-k} C_{q_{2}+L}^{k}=C_{K}^{q_{1}+L} C_{K}^{L}
$$

где $L=\left(K-q_{1}-q_{2}\right) / 2$ - половина числа поворотов. Тогда, пользуясь (31), получаем

$$
\mathcal{D}_{\lambda / 2}^{K}\left[F_{j_{1}, j_{2} ; l_{1}, l_{2}}(\lambda)\right]=\mathcal{T}_{K}\left(n_{11}, n_{22}\right)-\mathcal{T}_{K}\left(n_{21}, n_{12}\right)=\left|\begin{array}{cc}
C_{K}^{L} & C_{K}^{L+n_{21}} \\
C_{K}^{L} & C_{K}^{L+n_{11}}
\end{array}\right|,
$$

где $L=\left(K-n_{11}-n_{22}\right) / 2$ и используется равенство $n_{11}+n_{22}=n_{12}+n_{21}$. 


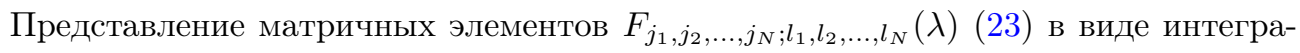
ла (24) позволяет получить следующее представление [15]:

$$
\begin{aligned}
F_{j_{1}, \ldots, j_{N} ; l_{1}, \ldots, l_{N}}(\lambda)= & \frac{e^{\lambda N}}{N !} \prod_{i=1}^{N}\left(\int_{-\pi}^{\pi} \frac{d \theta_{i}}{2 \pi}\right) e^{-\lambda \sum_{k=1}^{N}\left(1-\cos \theta_{k}\right)} \times \\
& \times S_{\boldsymbol{\pi}}\left(e^{i \theta_{1}}, e^{i \theta_{2}}, \ldots, e^{i \theta_{N}}\right) \prod_{1 \leqslant j<k \leqslant N}\left|e^{i \theta_{j}}-e^{i \theta_{k}}\right|^{2},
\end{aligned}
$$

где $S_{\boldsymbol{\pi}}\left(e^{i \theta_{1}}, e^{i \theta_{2}}, \ldots, e^{i \theta_{N}}\right)-$ функция Шура [24],

$$
S_{\boldsymbol{\pi}}\left(x_{1}, x_{2}, \ldots, x_{N}\right) \equiv \frac{\operatorname{det}\left(x_{j}^{\pi_{k}+N-k}\right)_{1 \leqslant j, k \leqslant N}}{\operatorname{det}\left(x_{j}^{N-k}\right)_{1 \leqslant j, k \leqslant N}} .
$$

Эта функция связана с разбиением $\boldsymbol{\pi}=\left(\pi_{1}, \pi_{2}, \ldots, \pi_{N}\right)$, определяемым последовательностью целых неотрицательных чисел, которые расположены в порядке нестрогого убывания: $\pi_{1} \geqslant \pi_{2} \geqslant \cdots \geqslant \pi_{N} \geqslant 0$. Благодаря трансляционной инвариантности всегда можно положить $l_{1}>l_{2}>\cdots>l_{N} \geqslant-N$ для начального положения пешеходов и задать элементы разбиения равенствами $\pi_{k}=l_{k}+k$. Для вычисления ведущей асимптотики производящей функции в пределе $\lambda \rightarrow \infty$ сведем интеграл (34) к следующему интегралу [7], [25]:

$$
\int d^{n} \theta \prod_{1 \leqslant j<k \leqslant N}\left|\theta_{j}-\theta_{k}\right|^{2} e^{-\lambda / 2 \sum_{k=1}^{N} \theta_{k}^{2}}=\frac{(2 \pi)^{N / 2}}{\lambda^{N^{2} / 2}}\left(\prod_{p=1}^{N} p !\right) .
$$

Это специальный случай интеграла Mexты, возникающего в теории гауссовых матричных ансамблей. В результате получаем асимптотику для производящей функции блужданий $N$ пешеходов:

$$
F_{j_{1}, \ldots, j_{N} ; l_{1}, \ldots, l_{N}}(\lambda) \simeq \mathcal{A} \frac{e^{\lambda N}}{\lambda^{N^{2} / 2}}, \quad \mathcal{A}=\frac{\prod_{p=1}^{N-1} p !}{(2 \pi)^{N / 2}} \prod_{1 \leqslant j<k \leqslant N} \frac{l_{j}-l_{k}}{k-j},
$$

где в $\mathcal{A}$ учтена известная формула для $S_{\boldsymbol{\lambda}}(1,1, \ldots, 1)$ [9], [25]. Таким образом, степенное поведение $F_{j_{1}, \ldots, j_{N} ; l_{1}, \ldots, l_{N}}(\lambda)$ определяется показателем $\xi=-N^{2} / 2$.

\section{2. Случайные блуждания над суперпозицией собственных состояний.} Собственные функции $X X$-гамильтониана, задаваемого соотношениями $(6),(7)$, строятся как комбинации состояний, которые возникают при "опрокидывании" $N$

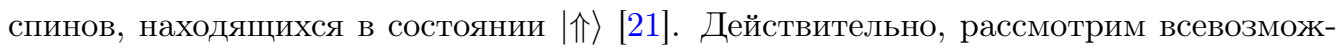
ные строгие разбиения $\boldsymbol{\mu}=\left(\mu_{1}, \mu_{2}, \ldots, \mu_{N}\right)$, где $M \geqslant \mu_{1}>\mu_{2}>\cdots>\mu_{N} \geqslant 1$. Каждому такому разбиению поставим в соответствие последовательность нулей и единиц вида $\left\{e_{k} \equiv e_{k}(\boldsymbol{\mu})\right\}_{k \in \mathcal{M}}$, где $e_{k}=\delta_{k, \mu_{n}}, 1 \leqslant n \leqslant N$. Искомую собственную функцию определим следующим образом:

$$
\left|\Psi_{N}\left(u_{1}, \ldots, u_{N}\right)\right\rangle=\sum_{\left\{e_{k}(\boldsymbol{\mu})\right\}_{k \in \mathcal{M}}} \Upsilon_{N}\left(\left\{u_{k}\right\} \mid \boldsymbol{\mu}\right)\left(\sigma_{M}^{-}\right)^{e_{M}}\left(\sigma_{M-1}^{-}\right)^{e_{M-1}} \ldots\left(\sigma_{1}^{-}\right)^{e_{1}}|\Uparrow\rangle,
$$


где в суммировании участвуют все строгие разбиения $\boldsymbol{\mu}$ указанного вида. Количество таких разбиений выражается числом перестановок $P(N, M-N)=C_{M}^{N}$ с повторениями (28). Для периодических граничных условий волновые функции

$$
\Upsilon_{N}\left(\left\{u_{k}\right\} \mid \boldsymbol{\mu}\right) \equiv \operatorname{det}\left(u_{k}^{2 \mu_{l}}\right)_{1 \leqslant k, l \leqslant N}
$$

параметризуются разбиениями $\boldsymbol{\mu}$ и различными, с точностью до перестановки, наборами $\left\{u_{1}, \ldots, u_{N}\right\}$ решений уравнений Бете:

$$
u_{k}^{2 M}=(-1)^{N-1}, \quad 1 \leqslant k \leqslant N .
$$

Эти решения имеют вид $u_{k}^{2}=e^{i 2 \pi I_{k} / M}$, где $I_{k}$ - это целые или полуцелые числа (в зависимости от четности $N$ ). В силу антисимметричности формулы (36) по отношению к перестановкам параметров $u_{k}$ достаточно ограничиться строгими разбиениями $M \geqslant I_{1}>I_{2}>\cdots>I_{N} \geqslant 1$. С учетом (36) мы получаем, что соответствующее нормированное среднее вида

$$
\left\langle\sigma_{m+1}^{+} e^{-\lambda H_{0}} \sigma_{1}^{-}\right\rangle_{N} \equiv \frac{\left\langle\Psi_{N}\left|\sigma_{m+1}^{+} e^{-\lambda H_{0}} \sigma_{1}^{-}\right| \Psi_{N}\right\rangle}{\left\langle\Psi_{N} \mid \Psi_{N}\right\rangle}
$$

может быть представлено как линейная комбинация $(N+1)$-точечных производящих функций (20) и, таким образом, связано с блужданиями $N+1$ пешехода. Начальное и конечные положения одного из них зафиксированы при $l_{1}=1$ и $j_{1}=m+1$, соответственно, в то время как для остальных (виртуалъных) пешеходов выбор положений случаен.

Представляет интерес вычисление среднего (39) в термодинамическом пределе, когда $M$ и $N$ растут (но их отношение остается конечным), т.е. число виртуальных пешеходов возрастает. В данном пределе [26]

$$
\left.\widetilde{F}_{m+1 ; 1}(\lambda) \equiv\left\langle\sigma_{m+1}^{+} e^{-\lambda H_{0}} \sigma_{1}^{-}\right\rangle_{N}\right|_{M, N \gg 1} \stackrel{\text { def }}{=} \operatorname{Tr}^{\prime}\left(\sigma_{m+1}^{+} e^{-\lambda H_{0}} \sigma_{1}^{-}\right),
$$

где $\operatorname{Tr}^{\prime}$ означает, что для вычисления нормированного среднего используется процедура, указанная в разделе 2 . Для $\widetilde{F}_{m+1 ; 1}(\lambda)(40)$ имеет место разностно-дифференциальное соотношение, аналогичное уравнению (18):

$$
\begin{aligned}
& \frac{d}{d \lambda} \widetilde{F}_{m+1 ; 1}(\lambda)=\frac{1}{2}\left(\widetilde{F}_{m ; 1}(\lambda)+\widetilde{F}_{m+2 ; 1}(\lambda)\right)-\operatorname{Tr}^{\prime}\left(H_{0} \sigma_{m+1}^{+} e^{-\lambda H_{0}} \sigma_{1}^{-}\right)- \\
& \quad-\operatorname{Tr}^{\prime}\left(\left(\frac{1-\sigma_{m+1}^{z}}{2}\right) \sigma_{m}^{+} e^{-\lambda H_{0}} \sigma_{1}^{-}\right)-\operatorname{Tr}^{\prime}\left(\left(\frac{1-\sigma_{m+1}^{z}}{2}\right) \sigma_{m+2}^{+} e^{-\lambda H_{0}} \sigma_{1}^{-}\right) .
\end{aligned}
$$

Вид данного уравнения заставляет предположить, что среднее $\widetilde{F}_{m+1 ; 1}(\lambda)$ тоже может представлять интерес как производящая функция случайных блужданий.

Займемся вычислением $\widetilde{F}_{m+1 ; 1}(\lambda)(40)$ в фермионном представлении (4). Удобно свести задачу к вычислению производящей функции следующего вида:

$$
\widetilde{\mathcal{G}} \equiv \operatorname{Tr}^{\prime}\left(e^{S} c_{m+1} e^{-\lambda H_{0}} c_{1}^{\dagger} e^{-\nu H_{0}}\right)
$$


где оператор $S$ определен так же, как и в (9) (т.е. с помощью матрицы $\widehat{S}=$ $\left.\operatorname{diag}\left\{S_{1}, S_{2}, \ldots, S_{M}\right\}\right)$. Действительно, $\widetilde{F}_{m+1 ; 1}(\lambda)$ соответствует выбору $\nu=0$ и $S_{k}=$ $-i \pi \theta(m-k)$, где $\theta(m-k)$ - функция Хевисайда, $\theta(0)=1$. Второе слагаемое в правой части (41) соответствует дифференцированию по $\nu$ в точке $\nu=0$, в третьем слагаемом мы считаем $\nu=0$ и дифференцируем по $S_{m+1}$. При этом в обоих случаях выбираем $S_{k}=-i \pi \theta(m-k)$. Учитывая, что вкладом слагаемых с индексом В в (11) можно пренебречь при достаточно больших $M$ и $N$, получаем приближенное равенство

$$
\begin{gathered}
\left.\widetilde{\mathcal{G}} \approx\left[\operatorname{tr}\left(e^{-\lambda \widehat{H}^{0}} \hat{e}_{1, m+1}\right)-\frac{d}{d \alpha}\right] \operatorname{det}\left(\hat{I}+\widehat{\mathcal{M}}_{1}+\alpha \widehat{\mathcal{M}}_{2}\right)\right|_{\alpha=0}, \\
\widehat{\mathcal{M}}_{1}+\alpha \widehat{\mathcal{M}}_{2} \equiv e^{-\nu \widehat{H}^{0}} e^{\widehat{S}} e^{-\lambda \widehat{H}^{0}}\left(\hat{I}+\alpha \hat{e}_{1, m+1} e^{-\lambda \widehat{H}^{0}}\right)
\end{gathered}
$$

где $\hat{e}_{1, m+1} \equiv\left(\delta_{1, n} \delta_{m+1, l}\right)_{1 \leqslant n, l \leqslant M}$. Матрица $\widehat{H}^{0}$ используется вместо $\widehat{H}^{ \pm}$, так как $s$ можно заменить нулем при достаточно больших $M$.

Соотношения (43) записаны в координатном представлении. Для перехода к импульсному представлению удобно воспользоваться некоторыми формулами, представленными в работе [22]. Ответ для $\widetilde{F}_{m+1 ; 1}(\lambda)$ запишем, сохраняя матричную символику (в пределе $M \rightarrow \infty$ соответствующие операции надо понимать в смысле действий над интегральными операторами [26]):

$$
\widetilde{F}_{m+1 ; 1}(\lambda)=\operatorname{det}\left(\hat{I}+\widehat{\mathcal{U}}_{m}\right)\left[\operatorname{tr}\left(e^{-\lambda \hat{\varepsilon}_{0}} \breve{e}_{1, m+1}\right)-\operatorname{tr}\left(\frac{\widehat{\mathcal{V}}_{m}}{\hat{I}+\widehat{\mathcal{U}}_{m}}\right)\right]
$$

(например, $\operatorname{tr}$ соответствует следу $(M \times M)$-матриц). Матрицы $\widehat{\mathcal{U}}_{m}, \widehat{\mathcal{V}}_{m}, \breve{e}_{1, m+1}$ соответствуют импульсным представлениям матриц $\widehat{\mathcal{M}}_{1}, \widehat{\mathcal{M}}_{2}, \hat{e}_{1, m+1}(43)$, но нам понадобятся только выражения для следов $\operatorname{tr} \widehat{\mathcal{U}}_{m}$ и $\operatorname{tr} \widehat{\mathcal{V}}_{m}$ (см. ниже). В импульсном представлении $\hat{\varepsilon}_{0}$ означает диагональную матрицу собственных значений энергии $X X$-модели при $h=0[21]$. Разложим формально $\widetilde{F}_{m+1 ; 1}(\lambda)$ по степеням $\widehat{\mathcal{U}}_{m}$ и получим ответ в двух низших порядках:

$$
\begin{aligned}
\widetilde{F}_{m+1 ; 1}(\lambda) & \approx F_{m+1 ; 1}(\lambda)+F_{m+1 ; 1}(\lambda) \operatorname{tr} \widehat{\mathcal{U}}_{m}-\operatorname{tr} \widehat{\mathcal{V}}_{m} \\
\operatorname{tr} \widehat{\mathcal{U}}_{m} & =(M-2 m) F_{1 ; 1}(\lambda) \\
\operatorname{tr} \widehat{\mathcal{V}}_{m} & =F_{m+1 ; 1}(2 \lambda)-2 \sum_{l=1}^{m} F_{m+1 ; l}(\lambda) F_{l ; 1}(\lambda)
\end{aligned}
$$

где обозначения $F_{j ; l}(\lambda)$ введены в $(24)$. Хотя $M$ и $m$ выбраны достаточно большими, отношение $m / M$ считается ограниченным. В каждом порядке уравнение (41) удовлетворяется представленными вкладами в $\widetilde{F}_{m+1 ; 1}(45)$.

Аналогично случаю ферромагнитного состояния подействуем оператором $\mathcal{D}_{\lambda / 2}^{K}$ на $\widetilde{F}_{m+1,1}(\lambda)(45)$. Тогда в первом порядке придем к соотношению $(26)$. Во втором порядке ответ имеет следующий вид:

$$
M C_{K}^{L} C_{K}^{L}-C_{K}^{L} \sum_{l=0}^{K} C_{K}^{l}+2 \sum_{l=1}^{m}\left|\begin{array}{cc}
C_{K}^{L+l-1} & C_{K}^{L} \\
C_{K}^{L} & C_{K}^{L}
\end{array}\right|
$$




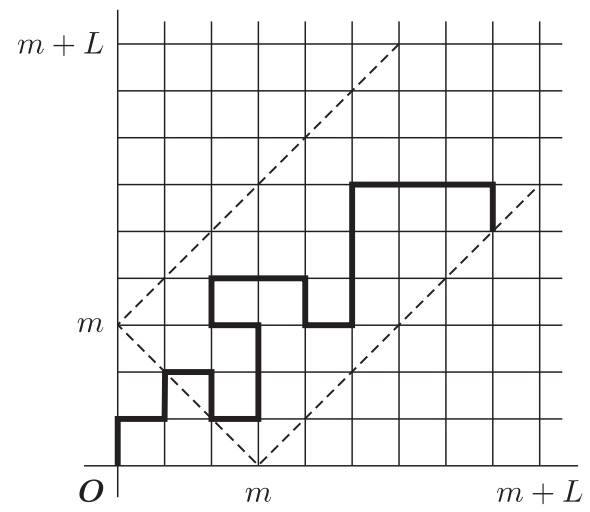

Типичная конфигурация.

В силу (33) результат применения $\mathcal{D}_{\lambda / 2}^{K}$ к функции второго порядка $F_{j_{1}, j_{2} ; l_{1}, l_{2}}(\lambda)$ связан, как частный случай $(31)$, с числом двумерных путей $\mathcal{T}_{K}$ и выражается через соответствующий определитель. Это означает, что уместно также представить (46) следующим эквивалентным образом:

$$
\begin{aligned}
& (M-K)\left|P_{K}(l \rightarrow l+m)\right|^{2}+ \\
& \quad+\mathcal{D}_{\lambda / 2}^{K}\left[2 \sum_{l=1}^{m}\left|\begin{array}{cc}
F_{m+1 ; l}(\lambda) & F_{m+1 ; 1}(\lambda) \\
F_{l ; l}(\lambda) & F_{l ; 1}(\lambda)
\end{array}\right|-\sum_{l=0}^{K}\left|\begin{array}{cc}
F_{m+L ; l}(\lambda) & F_{m+1 ; 1}(\lambda) \\
F_{l ; l}(\lambda) & F_{l ; L}(\lambda)
\end{array}\right|\right] .
\end{aligned}
$$

Другими словами, во втором порядке результат применения $\mathcal{D}_{\lambda / 2}^{K}$ к (45) можно переформулировать в терминах числа блужданий двух пешеходов (см. (23) и (33)) и квадрата числа блужданий одного пешехода. Суммирование по индексу $l$ в $\operatorname{tr} \widehat{\mathcal{V}}_{m}(45)$ можно интерпретировать как суммирование по положениям виртуального пешехода в (47).

Используя (33), можно представить (47) в терминах числа траекторий на двумерной решетке, это число равно

$$
(M-2(m+1)) \mathcal{T}_{K}(m, 0)+\sum_{l=0}^{m} \mathcal{T}_{K}(m-l, l)-\sum_{l=1}^{L} \mathcal{T}_{K}(m+l, l)-\sum_{l=1}^{L} \mathcal{T}_{K}(l, m+l)
$$

В этом выражении перечисляются решеточные траектории из $K$ звеньев, начинающиеся в точке $\boldsymbol{O}=(0,0)$ и заканчивающиеся на отрезках пунктирной ломаной, которая соединяет точки $(L, L+m),(0, m),(m, 0)$, и $(L+m, L)$ (см. рисунок). Формально сумма не является знакоопределенной, хотя ее асимптотика в целом положительна. Мы ожидаем, что аналогичное описание возможно и для более высоких порядков.

Оценим поведение числа путей, задаваемого представлением (46), в пределе, когда число звеньев $K=m+2 L$ возрастает. Будем считать, что выполняется ограничение $1 \ll m \ll L$. Иными словами, пусть $m$ растет умеренно по сравнению с ростом числа поворотов $L$, например пусть $L$ растет, как $m^{2}$. Пользуясь известным 
асимптотическим разложением логарифма гамма-функции [27], можно, в частности, оценить число сочетаний $C_{K}^{L}$. Ограничиваясь первым порядком малости, приходим к соотношению

$$
C_{K}^{L} \approx \frac{2^{K}}{\sqrt{\pi L}} e^{-m^{2} /(4 L)}\left(1-\frac{m}{2 L}\left(1-\frac{m^{2}}{4 L}\right)\right) \approx \frac{2^{K}}{\sqrt{\pi L}}\left(1-\frac{m^{2}}{4 L}\right) \sim \frac{2^{2 L}}{\sqrt{\pi L}} .
$$

Второе приближенное равенство в (49) имеет место, если $L$ растет быстрее, чем $m^{2}$. Оценка (49) характеризует рост числа траекторий (26) для одного пешехода.

Далее, третье слагаемое в (46) можно записать как $2 A(m, L) C_{K}^{L} C_{K}^{L}$, где

$$
A(m, L) \equiv-m+\sum_{l=1}^{m} \frac{(L+m+2-l)_{l-1}}{(L+1)_{l-1}}
$$

Здесь использовано стандартное обозначение для символа Похгаммера $(\alpha)_{n}[27]$. Пользуясь вновь разложением логарифма гамма-функции, мы можем оценить $A(m, L)(50)$ :

$$
A(m, L) \simeq m Z_{1}(m, L)-Z_{0}(m, L)+O\left(m^{-1}\right)
$$

где

$$
\begin{aligned}
& Z_{0}(m, L) \equiv e^{m^{2} / 4 L}\left(1+\frac{m}{L^{2}} \sum_{l=0}^{m / 2} e^{-l^{2} / L}\left(\frac{m^{2}}{4}-l^{2}\right)\right), \\
& Z_{1}(m, L) \equiv-1+e^{m^{2} / 4 L} \frac{2}{m} \sum_{l=0}^{m / 2} e^{-l^{2} / L}
\end{aligned}
$$

Пусть значения параметров $m$ и $L$ возрастают, но отношение $L / m^{2}$ остается ограниченным и имеет порядок единицы. Можно показать (включая численную проверку), что коэффициентные функции $Z_{0}(m, L)$ и $Z_{1}(m, L)$ остаются при этом ограниченными, и в $(51)$ вклад $Z_{0}(m, L)$ пренебрежим по сравнению с $m Z_{1}(m, L)$. Используем (49) и (51) для оценки величины (46) в ведущем приближении: имеем

$$
\frac{2^{4 L}}{\pi L} e^{-m^{2} / 2 L}\left(M+2 m Z_{1}(m, L)-(\pi L)^{1 / 2} e^{m^{2} / 4 L}\right) .
$$

Благодаря поведению коэффициента $Z_{1}(m, L)$ вклад соответствующего слагаемого в (53) может оказаться сопоставимым с $M$. Соотношение (53) свидетельствует о том, что картина блужданий в представлении суперпозиции собственных состояний сложнее, чем для ферромагнитного случая. Эта картина может рассматриваться как совместное блуждание исходного (основного) и виртуального пешеходов. Точки окончания траекторий, принадлежащие всем трем участкам пунктирной ломаной на рисунке (см. представление двумерных блужданий (48)), соответствуют сравнимым вкладам в оценку (53). В некоторых случаях, определяемых предельным поведением отношения $m^{2} / L$, доминирующим может оказаться вклад отрезка между точками $(m, 0)$ и $(0, m)$. 


\section{4. ЗАКЛЮЧЕНИЕ}

Показано, что корреляционные функции $X X$-магнетика Гейзенберга, вычисляемые над суперпозицией собственных состояний, как и для ферромагнитного случая, связаны с перечислением траекторий пешеходов, блуждающих по решетке. Установлена связь между числом траекторий нескольких недружественных пешеходов и числом путей единичного пешехода, перемещающегося со случайными поворотами по решетке, размерность которой совпадает с количеством недружественных пешеходов. Дифференцирование производящей функции, вычисленной для суперпозиции собственных состояний, демонстрирует более сложную комбинаторную картину, чем для ферромагнитного случая. В частности, к траекториям отдельного пешехода добавляются траектории, соответствующие совместному блужданию исходного и виртуального (недружественных) пешеходов. Получена оценка числа траекторий совместного блуждания основного и виртуального пешеходов.

Благодарности. Работа частично поддержана РФФИ (грант № 07-01-00358) и программой РАН "Математические методы нелинейной динамики".

\section{Список литературы}

[1] M. E. Fisher, J. Statist. Phys., 34:5-6 (1984), 667-729.

[2] P. J. Forrester, J. Phys. A, 23:7 (1990), 1259-1273.

[3] T. Nagao, P. J. Forrester, Nucl. Phys. B, 620:3 (2002), 551-565.

[4] A. J. Guttmann, A. L. Owczarek, X. G. Viennot, J. Phys. A, 31:40 (1998), 8123-8135.

[5] C. Krattenthaler, A. J. Guttmann, X. G. Viennot, J. Phys. A, 33:48 (2000), 8835-8866.

[6] C. Krattenthaler, A.J. Guttmann, X. G. Viennot, J. Statist. Phys., 110:3-6 (2003), 1069-1086.

[7] M. Katori, H. Tanemura, Phys. Rev. E, 66:1 (2002), 011105.

[8] M. Katori, H. Tanemura, T. Nagao, N. Komatsuda, Phys. Rev. E, 68:2 (2003), 021112.

[9] M. Katori, H. Tanemura, "Nonintersecting paths, noncolliding diffusion processes and representation theory", Combinatorial Methods in Representation Theory and their Applications, RIMS Kôkyûroku, 1438, ed. D. Sagaki, Res. Inst. Math. Sci., Kyoto, 2005, 83-102.

[10] P. Bak, C. Tang, K. Wiesenfeld, Phys. Rev. A, 38:1 (1988), 364-374.

[11] D. Huse, M. Fisher, Phys. Rev. B, 29:1 (1984), 239-270.

[12] J. W. Essam, A. J. Guttmann, Phys. Rev. E, 52:6 (1995), 5849-5862.

[13] С. Ю. Григорьев, В. Б. Приезжев, ТМФ, 146:3 (2006), 488-498.

[14] J. W. van de Leur, A. Yu. Orlov, Random turn walk on a half line with creation of particles at the origin, arXiv: 0801.0066.

[15] Н. М. Боголюбов, Зап. научн. сем. ПОМИ, 325, 2005, 13-27.

[16] Н. М. Боголюбов, Зап. научн. сем. ПОМИ, 335, 2006, 59-74.

[17] C. Malyshev, "Functional integration with "automorphic" boundary conditions and correlators of $z$-components of spins in the $X Y$ and $X X$ Heisenberg chains", New Developments in Mathematical Physics Research, ed. C. V. Benton, Nova Sci., New York, 2004, 85-116.

[18] К. Л. Малышев, Зап. научн. сем. ПОМИ, 317, 2004, 142-173.

[19] E. Lieb, T. Schultz, D. Mattis, Ann. Physics, 16:3 (1961), 407-466.

[20] Th. Niemeijer, Physica, 36:3 (1967), 377-419; 39:3 (1968), 313-326.

[21] Ф. Коломо, А. Г. Изергин, В. Е. Корепин, В. Тогнетти, ТМФ, 94:1 (1993), 19-51.

[22] А. Г. Изергин, В. С. Капитонов, Н. А. Китанин, Зап. научн. сем. ПОМИ, 245, 1997, 173-206.

[23] В. С. Капитонов, А. Г. Пронько, Зап. научн. сем. ПОМИ, 269, 2000, 219-261. 
[24] Э. Прессли, Г. Сигал, Группы петель, Мир, М., 1990.

[25] M. Katori, H. Tanemura, Scaling limit of vicious walkers, Schur function, and Gaussian random matrix ensemble, arXiv: cond-mat/0110274.

[26] V. E. Korepin, N. M. Bogoliubov, A. G. Izergin, Quantum Inverse Scattering Method and Correlation Functions, Cambridge Monogr. Math. Phys., Cambridge Univ. Press, Cambridge, 1993.

[27] W. Magnus, F. Oberhettinger, R. P. Soni, Formulas and Theorems for the Special Functions of Mathematical Physics, Grundlehren Math. Wiss., 52, Springer, New York, 1966. 